\title{
Produção de óleos essenciais em plantas de Mentha $x$ piperita L. var. piperita (Lamiaceae) submetidas a diferentes níveis de luz e nutrição do substrato ${ }^{1}$
}

\author{
ROQUE L. PEGORARO², MIRIAM DE B. FALKENBERG ${ }^{3}$, CAROLINE H. VOLTOLINI $^{2}$, \\ MARISA SANTOS ${ }^{2}$ e MARIA TEREZINHA S. PAULILO ${ }^{2,4}$
}

(recebido: 17 de janeiro de 2008; aceito: 21 de outubro de 2010)

\begin{abstract}
Production of essential oils in plants of Mentha x piperita L. var. piperita (Lamiaceae) submitted to different light levels and nutrition of the substratum). Mentha x piperita L. var. piperita (mint pepper) is utilized due to the presence of essential oils, mainly because of the component menthol produced in glandular trichomes. The influence of light intensity and substratum fertility was evaluated on the amount produced and quality of the essential oil. The light intensities utilized were $100 \%, 70 \%$ and $50 \%$ of the total solar light and two levels of nutrition of the substratum were applied (forest soil and forest soil with addition of organic fertilizer. The high light intensity and the addition of fertilizer favored the growth in biomass, influencing in this sense the production of the essential oil per plant. The light intensity and the addition of fertilizer influenced the oil quality, showing the plants under full sun light and fertilization with higher menthol content than plants in shadow or without fertilization. Menthol was the major component of the essential oil.
\end{abstract}

Key words - essential oils, growth, light intensity, organic fertilizer

RESUMO - (Produção de óleos essenciais em plantas de Mentha x piperita L. var. piperita (Lamiaceae) submetidas a diferentes níveis de luz e nutrição do substrato). Mentha x piperita L. var. piperita (hortelã-pimenta) é bastante utilizada devido à presença de óleos essenciais, principalmente pelo componente mentol, produzidos nos tricomas glandulares. Foi avaliada a influência da intensidade de luz e da adubação do substrato na quantidade e qualidade do óleo essencial. As intensidades de luz utilizadas foram $100 \%, 70 \%$ e $50 \%$ da luz solar total e dois níveis de nutrição do substrato aplicados, solo de mata e solo de mata com adição de adubo orgânico. A alta intensidade de luz e a adubação favoreceram o crescimento em biomassa, influenciando no rendimento do óleo essencial por planta. A intensidade de luz e a adubação influenciaram na qualidade do óleo essencial, apresentando as plantas sob luz solar plena e adubadas apresentaram maior concentração relativa de mentol que plantas sombreadas ou sem adubo. O mentol foi o componente majoritário encontrado no óleo essencial.

Palavras-chave - adubação, crescimento, intensidade de luz, óleos essenciais

\section{Introdução}

Mentha $x$ piperita L. var. piperita (Lamiaceae) é um híbrido estéril, produto do cruzamento entre M. spicata L. e M. aquatica L. (Sato et al. 1996), muito utilizado para fins medicinais, alimentícios e cosméticos (Cardoso et al. 2001) devido à presença de óleos essenciais produzidos nos tricomas glandulares, principalmente nos peltados (Turner et al.2000). O óleo essencial dessa espécie é constituído principalmente por

1. Parte da dissertação de mestrado do primeiro autor, Programa de Pós-Graduação em Biologia Vegetal, Universidade Federal de Santa Catarina, SC, Brasil..

2. Universidade Federal de Santa Catarina, Departamento de Botânica, Campus Universitário Trindade, 88040-970 Florianópolis, SC, Brasil.

3. Universidade Federal de Santa Catarina, Programa de Pós-Graduação em Farmácia, Campus Universitário Trindade, 88040-970 Florianópolis, SC, Brasil.

4. Autor para correspondência: paulilo@ccb.ufsc.br monoterpenos, atribuindo-se a estes as funções de defesa da planta contra herbivoria, agentes antimicrobianos e alelopáticos (Cardoso et al. 2001). O mentol e a mentona são os principais componentes do óleo e os de maior valor econômico, embora sejam conhecidos mais de 200 componentes presentes nos óleos do gênero Mentha (Tavish \& Harris 2002). Fatores ambientais e a fase de desenvolvimento da planta podem influenciar na qualidade e quantidade de óleos essenciais (Rodrigues et al. 2004, Rohloff et al. 2005, David et al. 2006). A intensidade luminosa, o fotoperíodo, a temperatura e a nutrição do solo podem influenciar diretamente na produção do óleo, ou indiretamente, através do aumento de biomassa das plantas de espécies produtoras de óleos essenciais (Lima et al. 2003, Rodrigues et al. 2004, Valmorbida et al. 2006). No caso de Menta x piperita, embora vários trabalhos tenham analisado o papel de nutrientes minerais (Praszna et al. 1973, David et al. 2006) e o da intensidade luminosa (Tavish \& Harris 
2002, Lima et al. 2003), no crescimento da planta e na produção de óleos essenciais, a investigação do efeito conjunto destes dois fatores ambientais sobre tais aspectos e em especial sobre a produção de mentol carece de dados. Desta maneira, o presente trabalho visa avaliar em Mentha x piperita a influência conjunta da intensidade de luz e da disponibilidade nutricional sobre a produção de óleos essenciais, bem como sobre aspectos da morfologia e crescimento da planta, os quais podem afetar indiretamente a produção destes óleos.

\section{Material e Métodos}

O material de estudo, Mentha x piperita L. var. piperita, foi identificado pelo Dr. Ray Harley (Royal Botanic Gardens, Kew - Reino Unido). A exsicata foi depositada no Herbário FLOR da Universidade Federal de Santa Catarina sob o no 35851 .

O cultivo de plantas foi conduzido em área aberta da Universidade Federal de Santa Catarina, Florianópolis, SC $\left(270^{\circ} 35^{\prime} 36^{\prime \prime}\right.$ S e $\left.480^{\circ} 35^{\prime} 60^{\prime \prime} \mathrm{W}\right)$, no período de 13 de agosto de 2005 a 31 de janeiro de 2006. As temperaturas médias mensais no período de cultivo, fornecidas pela Epagri, SC, oscilaram entre $17,22^{\circ} \mathrm{C}$ até $25,89^{\circ} \mathrm{C}$. As mudas foram produzidas através de segmentos de caules enterrados na areia e quando apresentavam três a quatro pares de folhas foram transplantadas para sacos plásticos com volume de $2 \mathrm{~L}$, contendo terra (coletada em região de mata) e composto orgânico na proporção de 1:1 (substrato adubado) ou apenas terra (substrato não adubado). $\mathrm{O}$ adubo orgânico utilizado foi húmus, marca Aduplan ${ }^{\circledR}$. As características dos substratos utilizados, fornecidas pela CIDASC, foram para substrato adubado: $\mathrm{pH}-6,4 ; P>50,0 \mathrm{ppm} ; \mathrm{K}-1064,0 \mathrm{ppm}$, Matéria orgânica $-9,6 \%$; $\mathrm{Ca}-7,50$ cmolc L $^{-1} ; \mathrm{Mg}-7,90$ cmolc L $^{-1} \mathrm{e}$ para substrato sem acréscimo de adubo: $\mathrm{pH}-5,6 ; \mathrm{P}-6,70 \mathrm{ppm}$; $\mathrm{K}-92,00$ ppm; Matéria orgânica $-6,5 \%$; $\mathrm{Ca}-7,00 \mathrm{cmolc} \mathrm{L}^{-1}$; $\mathrm{Mg} 5,00 \mathrm{cmolc} \mathrm{L}^{-1}$. O experimento constituiu-se de quatro tratamentos: plantas em substrato adubado a pleno sol (T1), a $70 \%$ de luz (T2) e a 50\% de luz (T3) e plantas em substrato não adubado a pleno sol (T4). A redução da intensidade de luz foi dada por tela de polietileno preta (sombrite) de diferentes malhas, presa à armação de madeira de $1 \mathrm{~m}^{3}$. A intensidade de luz solar plena, medida com quantômero Licor 250 ao meio dia de um dia claro, foi de $1800 \mu \mathrm{mol}$ de fótons $\mathrm{m}^{-2} \mathrm{~s}^{-1}$, a $70 \%$ de luz foi de $1250 \mu \mathrm{mol}$ de fótons $\mathrm{m}^{-2} \mathrm{~s}^{-1}$ e a $50 \%$ de luz foi de $900 \mu \mathrm{mol}$ de fótons $\mathrm{m}^{-2} \mathrm{~s}^{-1}$. Cada tratamento foi composto de três unidades experimentais, cada unidade contendo cinco plantas. As plantas foram irrigadas em dias alternados. Entre 100 e 130 dias após o transplante, três plantas por tratamento foram coletadas para determinação da massa seca, densidades estomáticas e de tricomas glandulares peltados e capitados. Aos 140 dias pós-transplante, de nove plantas por tratamento foram coletadas folhas adultas e jovens para a extração dos óleos essenciais. Nesta época as plantas iniciaram seu florescimento, à exceção das plantas não adubadas.
Os dados encontrados foram submetidos à análise de variância (ANOVA) para comparação de mais de duas médias, seguida do teste de Tukey. Quando foram comparadas duas médias, utilizou-se o teste $t$ (Sokal \& Rohlf 1969). O nível de confiabilidade foi de $95 \%$. A análise foi feita através do programa computacional STATISTICA 6.0.

A massa seca foi determinada por pesagem após a secagem do material por 48 horas a $80^{\circ} \mathrm{C}$. Raízes e partes aéreas foram pesadas, separadamente, em balança analítica. A área foliar individual foi determinada com folhas da porção média do caule, através da comparação do peso do contorno das folhas desenhadas em papel com o peso de áreas conhecidas do mesmo papel. Foram utilizadas três plantas por tratamento, sendo que de cada planta retiraram-se nove folhas adultas de vários ramos, num total de vinte e sete folhas por tratamento. A área foliar total das folhas adultas coletadas foi determinada pela multiplicação da área média individual de folhas adultas pelo número de folhas adultas.

Foram calculadas a razão raiz/parte aérea (R/PA), a massa foliar específica (MFE) e a taxa média de crescimento relativo (TCR) segundo Hunt (1982). R/PA = Mraiz/Mparte aérea, MFE $=$ Mfoliar/A e TCR $=(\ln \mathrm{M} 2-\ln \mathrm{M} 1) /(\mathrm{T} 2-\mathrm{T} 1)$, onde $\mathrm{M} 2$ = massa seca da planta da coleta $2, \mathrm{M} 1=$ massa seca da planta da coleta $1, \mathrm{M}=$ massa seca, $\mathrm{T} 1=$ tempo da primeira coleta, $\mathrm{T} 2$ = tempo da segunda coleta, $\mathrm{A}=$ área foliar.

Para a determinação da densidade estomática e de tricomas, impressões da epiderme da região mediana das faces abaxial foram feitas com esmalte incolor de unhas, e porções de películas de esmalte retiradas foram montadas em lâmina e lamínula. Para a contagem de tricomas e estômatos utilizaram-se folhas jovens (as inseridas no terceiro nó caulinar basípeto) e folhas adultas (inseridas logo abaixo do terceiro nó) de três plantas por tratamento, três folhas de cada grau de desenvolvimento por planta. As contagens dos estômatos e tricomas foram feitas utilizando-se microscópio óptico, marca Zeiss modelo Laboval, fazendo leitura direta de 27 campos em aumento de $400 \mathrm{X}$ (estômatos e tricomas capitados) e de $100 \mathrm{X}$ (tricomas peltados). O diâmetro do campo circular foi calculado com o auxílio de uma lâmina marcada em escala micrométrica.

A determinação das dimensões das células-guarda foi feita considerando o comprimento (eixo longitudinal, entre os dois pólos da célula) e largura (eixo transversal, na porção média da célula), quando em vista frontal da superfície epidérmica. As imagens foram projetadas sobre papel, com auxílio de câmara clara acoplada ao microscópio óptico. As dimensões foram determinadas com régua milimetrada e aferidas com escala micrometrada, para apresentação dos dados em micrômetros.

Para a extração dos óleos essenciais, as plantas foram colhidas no início da manhã. Folhas jovens e adultas foram separadas e acondicionadas em sacos plásticos e mantidas em geladeira até a extração dos óleos. Cada amostra, correspondendo a folhas de três plantas por tratamento, foi pesada e submetidas à extração do óleo por arraste de vapor d'água durante uma hora com o auxílio do aparelho 
de Clevenger (Farmacopéia Brasileira 2000). O óleo volátil extraído foi acondicionado em vidro âmbar devidamente etiquetado e dessecado com $\mathrm{Na}_{2} \mathrm{SO}_{4}$ anidro e guardado em geladeira (Simões \& Sptizer 2004).

A análise semi-quantitativa dos óleos foi feita por cromatografia em camada delgada (CCD) para todas as amostras de óleo juntamente com um padrão de mentol de concentração conhecida. Os óleos essenciais e o padrão de mentol foram dissolvidos em três partes $(\mathrm{v} / \mathrm{v})$ de clorofórmio e aplicados com capilares padronizados em placas de sílica GF254, eluídas em clorofórmio/éter de petróleo (1:1). As placas foram reveladas com anisaldeído-sulfúrico a $100{ }^{\circ} \mathrm{C}$ e observadas sob luz UV 254 e $365 \mathrm{~nm}$ (Stahl \& Schild 1981). Para documentação, as placas foram digitalizadas em scanner marca Genius - ColorPage-Vivid III V2. As áreas das manchas correspondentes ao mentol foram medidas através de planímetro digital PLACON KP $-80 \mathrm{~N}$.

O rendimento relativo do óleo foi calculado em $\mathrm{mL}$ por $100 \mathrm{~g}$ de massa fresca para folhas jovens e para folhas adultas. Também foi calculado o rendimento de óleo em folhas adultas em $\mathrm{mL}$ por 1000 tricomas e em $\mathrm{mL}$ por $\mathrm{cm}^{2}$.

\section{Resultados e Discussão}

Foram encontrados em Mentha x piperita L. var. piperita, tricomas glandulares peltados e capitados nas faces da epiderme adaxial e abaxial das folhas e nos caules. Não foram encontrados tricomas não glandulares, embora Turner et al. (2000) relate a presença em $M$. $x$ piperita de tricomas não-glandulares unicelulares e multicelulares unisseriados. As folhas apresentaram estômatos nas duas epidermes, do tipo diacítico de acordo com a classificação de Wilkinson (1988).

A intensidade de luz e a nutrição do substrato influenciaram a biomassa das plantas (tabela 1). O aumento em biomassa, tanto em parte aérea como em raiz, foi proporcional ao aumento da intensidade de luz, reflexo do aumento da taxa de crescimento relativo (TCR). O aumento de intensidade luminosa geralmente causa um aumento do consumo de água, mas as plantas apresentaram capacidade de ajustar-se a esta situação, aumentando a proporção de biomassa entre raiz e parte aérea (R/PA), o que favorece a captação de água e nutrientes (Poorter 1999) e aumentando a relação entre massa e área foliar (MFE), o que contribui para diminuição da transpiração (Castro et al. 2007).

A adubação proporcionou um aumento de biomassa, resultado do aumento da TCR. Proporcionou também aumento da área foliar, sendo a área foliar média individual duas vezes maior, e a totais nove vezes maior que a das plantas não adubadas. A proporção de massa foliar sobre área foliar (MFE) aumentou em plantas adubadas, indicando maior alocação de biomassa por área. Um aumento nesta proporção pode

Tabela 1. Massa seca total (Mtotal), de raiz (MRaiz) e de parte aérea (MPA), razão raiz/parte aérea (R/PA), massa foliar específica (MFE), taxa de crescimento relativo (TCR), área foliar (AF) individual e total de folhas adultas de Mentha x piperita L. var. piperita cultivadas sob condições diferentes de luz e nutrição do substrato.

Table 1. Total dry mass (MTotal), root/shoot ratio (R/PA), specific leaf mass (MFE), relative growth rate (TCR), individual and total leaf area (AF) of Mentha x piperita L. var. piperita cultivated under different conditions of light and nutrition of the substratum.

\begin{tabular}{|c|c|c|c|c|c|c|c|c|}
\hline Tratamentos & $\begin{array}{l}\text { Mtotal } \\
(\mathrm{g})\end{array}$ & $\begin{array}{l}\text { Raiz } \\
(\mathrm{g})\end{array}$ & $\begin{array}{l}\text { PA } \\
(g)\end{array}$ & $\mathrm{R} / \mathrm{PA}$ & $\begin{array}{c}\text { MFE } \\
\left(\mathrm{mg} \mathrm{cm}^{-2}\right)\end{array}$ & $\begin{array}{c}\text { TCR } \\
\mathrm{mg} \mathrm{mg}^{-1} \mathrm{~d}^{-1}\end{array}$ & $\begin{array}{l}\text { AF individual } \\
\text { média }\left(\mathrm{cm}^{2}\right)\end{array}$ & $\begin{array}{l}\text { AF total } \\
\left(\mathrm{cm}^{2}\right)\end{array}$ \\
\hline $100 \%$ luz & $\begin{array}{l}24,4 \mathrm{a} \\
( \pm 2,9)\end{array}$ & $\begin{array}{c}5,1 \mathrm{a} \\
( \pm 1,3)\end{array}$ & $\begin{array}{l}19,0 \mathrm{a} \\
( \pm 2,1)\end{array}$ & $\begin{array}{c}0,3 \mathrm{a} \\
( \pm 0,06)\end{array}$ & $\begin{array}{c}5,9 \mathrm{a} \\
( \pm 0,3)\end{array}$ & $\begin{array}{c}0,067 \mathrm{a} \\
( \pm 0,001)\end{array}$ & $\begin{array}{c}7,5 \mathrm{~b} \\
( \pm 0,27)\end{array}$ & $\begin{array}{l}6466 \mathrm{a} \\
( \pm 639)\end{array}$ \\
\hline $70 \%$ luz & $\begin{array}{l}16,9 \mathrm{ab} \\
( \pm 2,4)\end{array}$ & $\begin{array}{l}4,2 \mathrm{ab} \\
( \pm 0,3)\end{array}$ & $\begin{array}{l}12,7 \mathrm{~b} \\
( \pm 2,2)\end{array}$ & $\begin{array}{c}0,3 \mathrm{a} \\
( \pm 0,04)\end{array}$ & $\begin{array}{c}4,5 \mathrm{~b} \\
( \pm 0,06)\end{array}$ & $\begin{array}{l}0,063 \mathrm{ab} \\
( \pm 0,033)\end{array}$ & $\begin{array}{c}9,1 \mathrm{a} \\
( \pm 0,12)\end{array}$ & $\begin{array}{l}5022 \mathrm{~b} \\
( \pm 157)\end{array}$ \\
\hline $50 \%$ luz & $\begin{array}{l}14,6 \mathrm{~b} \\
( \pm 3,3)\end{array}$ & $\begin{array}{c}2,6 \mathrm{~b} \\
( \pm 0,8)\end{array}$ & $\begin{array}{l}12,0 \mathrm{~b} \\
( \pm 2,5)\end{array}$ & $\begin{array}{c}0,2 \mathrm{~b} \\
( \pm 0,03)\end{array}$ & $\begin{array}{l}4,1 \mathrm{~b} \\
( \pm 0,3)\end{array}$ & $\begin{array}{c}0,062 \mathrm{~b} \\
( \pm 0,002)\end{array}$ & $\begin{array}{c}9,6 \mathrm{a} \\
( \pm 1,0)\end{array}$ & $\begin{array}{l}5245 \mathrm{ab} \\
( \pm 621)\end{array}$ \\
\hline Sem adubo & $\begin{array}{c}5,2 \mathrm{~B} \\
( \pm 3,3)\end{array}$ & $\begin{array}{l}1,4 \mathrm{~B} \\
( \pm 0,6)\end{array}$ & $\begin{array}{l}4,1 \mathrm{~B} \\
( \pm 2,7)\end{array}$ & $\begin{array}{c}0,3 \mathrm{~A} \\
( \pm 0,09)\end{array}$ & $\begin{array}{l}4,5 \mathrm{~B} \\
(0,6)\end{array}$ & $\begin{array}{c}0,050 \mathrm{~B} \\
( \pm 0,005)\end{array}$ & $\begin{array}{l}3,4 \mathrm{~B} \\
( \pm 0,7)\end{array}$ & $\begin{array}{l}681 \mathrm{~B} \\
( \pm 195)\end{array}$ \\
\hline Com adubo & $\begin{array}{l}24,4 \mathrm{~A} \\
( \pm 2,9)\end{array}$ & $\begin{array}{c}5,1 \mathrm{~A} \\
( \pm 1,3)\end{array}$ & $\begin{array}{l}19,0 \mathrm{~A} \\
( \pm 2,1)\end{array}$ & $\begin{array}{c}0,3 \mathrm{~A} \\
( \pm 0,06)\end{array}$ & $\begin{array}{c}5,9 \mathrm{~A} \\
( \pm 0,3)\end{array}$ & $\begin{array}{l}0,0667 \mathrm{~A} \\
( \pm 0,001)\end{array}$ & $\begin{array}{c}7,5 \mathrm{~A} \\
( \pm 0,27)\end{array}$ & $\begin{array}{l}6466 \mathrm{~A} \\
( \pm 639)\end{array}$ \\
\hline
\end{tabular}

Valores seguidos de letras minúsculas distintas diferem nas colunas para os tratamentos de luz, sendo $P<0,05$ pela ANOVA seguida de teste de Tukey. Valores seguidos de letras maiúsculas distintas diferem pelo teste $t$ (Student) onde $P<0,05$. ( \pm ) desvio padrão da média / Numbers followed by different letters in the columns differ significantly by ANOVA test where $P<0.05$ (minor letters) and in lines (major letters) by test $\mathrm{t}$ (Student) where $P<0.05$. ( \pm ) standard deviation.. 
ser devido ao alongamento e ou adição de células do parênquima paliçádico (Poorter 1999). Essa alteração do aparato fotossintético favorece a fotossíntese (Chazdon \& Kaufmann 1993) e poderá acarretar alterações no metabolismo secundário (Castro et al. 2005).

Os resultados encontrados para adição de adubação concordam com os resultados de outros trabalhos com Mentha x piperita, os quais verificaram que na ausência de nitrogênio, fósforo e potássio a massa total e a massa foliar das plantas foram reduzidas (Praszna \& Bernáth 1993), que baixos níveis de potássio reduziram a TCR e massa seca (Valmorbida 2006) e que a redução de fósforo levou à redução de massa seca e fresca (Rodrigues et al. 2004). As plantas cultivadas com adubo iniciaram o seu florescimento aos 140 dias após o replantio e as não adubadas não floresceram até o término do experimento, dados que concordam com os de Ghosh \& Chatierjee (1976) para M. x piperita, que encontraram florescimento mais rápido em plantas de solos bem nutridos em relação àquelas cultivadas em solos mais pobres.

Em Mentha x piperita, a densidade estomática (tabela 2) foi maior para as plantas cultivadas em intensidade de luz mais alta, mas o número total de estômatos por folha não variou em função da intensidade luminosa, indicando que o aumento da densidade estomática ocorrida deve ter sido causado pela menor expansão das células epidérmicas, tornando os estômatos mais próximos entre si, como também verificado por Knecht \& O'Leary (1972) e Silva \& Anderson (1985) para a herbácea Phaseolus vulgaris L. Variação na densidade estomática em função do ambiente é importante na regulação de trocas gasosas de plantas (Castro et al. 2005). O aumento da densidade estomática em alta intensidade luminosa pode contribuir para a diminuição da saída de vapor de água, mas não para a entrada e gás carbônico (Larcher 2002), aumentando a eficiência na utilização de água em relação à fotossíntese na planta (Medri \& Lledras 1980, Naves et al. 1994). Em plantas adubadas, a densidade estomática foi similar às não adubadas, mas o número de estômatos por folha foi maior em plantas adubadas, indicando que a adubação aumentou a diferenciação das células epidérmicas em novos estômatos. Estes dados concordam com aqueles obtidos por Jurik et al. (1982) para Fragaria virginiana. $\mathrm{O}$ aumento do número de estômatos com a adubação favorece a fotossíntese pela facilitação na difusão de gás carbônico (Melo et al. 2007). A variação do comprimento e largura das células-guarda em função de alteração do ambiente é uma característica dependente da espécie (Castro et al. 2005) e no caso de Mentha $x$ piperita estas características não foram afetadas pela intensidade de luz ou adubação (tabela 2). A densidade de tricomas em folhas adultas não foi influenciada pela intensidade de luz, mas foi pela adubação, apresentando maior densidade de tricomas a folha de plantas adubadas (tabela 3). As folhas jovens apresentaram, na maior parte dos tratamentos, densidade de estômatos e tricomas maior que em folhas adultas (tabela 3), resultado similar ao encontrado por Maffei et al. (1989) e Turner et al. (2000) para esta mesma espécie. Este resultado pode ser conseqüência da expansão incompleta das células (Wilkinson 1988).

A intensidade de luz e a adubação não influenciaram no rendimento de óleo por tricomas ou por $\mathrm{cm}^{2}$ de área foliar (tabela 4). Duriyaprapan et al. (1986) também encontraram resultados semelhantes em Mentha arvensis.

Tabela 2. Densidade estomática, número de estômatos por folha adulta, comprimento e largura das células-guarda estomáticas de Mentha x piperita var. piperita cultivadas sob condições diferentes de luz e nutrição do substrato.

Table 2. Stomatal density, number of stomata per leaf, length and width of the stomatal cell-guard of Menthax piperita L. var. piperita cultivated under different conditions of light and nutrition of the substratum.

\begin{tabular}{|c|c|c|c|c|}
\hline \multirow[t]{2}{*}{ Tratamento } & Densidade estomática & $\begin{array}{c}\text { № de estômatos } \\
\text { por folha }\end{array}$ & $\begin{array}{l}\text { Comprimento da } \\
\text { célula guarda }\end{array}$ & $\begin{array}{l}\text { Largura da } \\
\text { célula guarda }\end{array}$ \\
\hline & $\left(\mathrm{n}^{\mathrm{o}} \mathrm{mm}^{-2}\right)$ & $\left(\times 10^{2}\right)$ & $(\mu \mathrm{m})$ & $(\mu \mathrm{m})$ \\
\hline $100 \%$ luz & 328,56 a $( \pm 45)$ & 2475 a $( \pm 89)$ & $26,50 \mathrm{a}( \pm 2,2)$ & $6,91 \mathrm{a}( \pm 1,2)$ \\
\hline $70 \%$ luz & 317,36 a $( \pm 39)$ & 2899 a $( \pm 38)$ & 25,14 a $( \pm 2,9)$ & $7,62 \mathrm{a}( \pm 1,5)$ \\
\hline $50 \%$ luz & 282,26 b $( \pm 39)$ & 2700 a $( \pm 295)$ & $26,78 \mathrm{a}( \pm 2,8)$ & $6,98 \mathrm{a}( \pm 1,2)$ \\
\hline Sem adubo & $312,88 \mathrm{~A}( \pm 40)$ & $954 \mathrm{~B}( \pm 235)$ & $26,49 \mathrm{~A}( \pm 2,7)$ & $6,91 \mathrm{~A}( \pm 1,1)$ \\
\hline Com adubo & $328,56 \mathrm{~A}( \pm 45)$ & $2475 \mathrm{~A}( \pm 89)$ & $27,14 \mathrm{~A}( \pm 2,2)$ & $7,26 \mathrm{~A}( \pm 1,2)$ \\
\hline
\end{tabular}

Valores seguidos de letras minúsculas distintas diferem nas colunas para os tratamentos de luz, com $P<0,05$ pela ANOVA seguida do teste de Tukey. Valores seguidos de letras maiúsculas distintas nas colunas diferem pelo teste $t$ (Student) onde $P<0,05$. ( \pm ) desvio padrão da média / Numbers followed by different letters in the columns differ significantly by ANOVA test where $P<0.05$ (small letters) and in lines (capital letters) by t test (Student) where $P<0.05$. ( \pm ) standard deviation. 
Tabela 3. Densidade de tricomas glandulares e de estômatos em folhas jovens e adultas de Mentha x piperita var. piperita cultivadas sob condições diferentes de luz e nutrição dos substratos. $(\mathrm{Fj}=$ folha jovem; $\mathrm{Fa}=$ folha adulta; $\mathrm{Cj}=$ caule jovem; $\mathrm{Ca}=$ caule adulto).

Table 3. Glandular trichomes and stomatal density of young and mature leaves of Mentha x piperita L. var. piperita cultivated under different conditions of light and nutrition of the substratum. ( $\mathrm{Fj}=$ young leaf; $\mathrm{Fa}=$ adult leaf; $\mathrm{Cj}=$ young stem; $\mathrm{Ca}=$ adult stem).

\begin{tabular}{|c|c|c|c|c|c|c|}
\hline \multirow{3}{*}{ Tratamentos } & \multicolumn{4}{|c|}{$\begin{array}{l}\text { Densidade de tricomas } \\
\qquad\left(\mathrm{n}^{\mathrm{o}} \mathrm{mm}^{-2}\right)\end{array}$} & \multirow{2}{*}{\multicolumn{2}{|c|}{$\begin{array}{c}\begin{array}{c}\text { Densidade estomática } \\
\left(\mathrm{n} \cong \mathrm{m} m^{-2}\right)\end{array} \\
\text { FOLHAS } \\
\end{array}$}} \\
\hline & \multicolumn{2}{|c|}{ FOLHAS } & \multicolumn{2}{|c|}{ CAULES } & & \\
\hline & $\mathrm{Fj}$ & $\mathrm{Fa}$ & $\mathrm{Cj}$ & $\mathrm{Ca}$ & $\mathrm{Fj}$ & $\mathrm{Fa}$ \\
\hline $100 \%$ de luz & 86,03 aA $( \pm 17)$ & $59,27 \mathrm{aB}( \pm 16)$ & $111,16 \mathrm{abA}( \pm 18)$ & 56,98 bB $( \pm 9)$ & $356,93 \mathrm{aA}( \pm 35)$ & $328,56 \mathrm{aB}( \pm 45)$ \\
\hline $70 \%$ de luz & $73,6 \mathrm{bA}( \pm 21)$ & $60,66 \mathrm{aB}( \pm 13)$ & $95,8 \mathrm{bA}( \pm 22)$ & 57,74 bB $( \pm 11)$ & $343,49 \mathrm{aA}( \pm 46)$ & $317,36 \mathrm{aB}( \pm 39)$ \\
\hline $50 \%$ de luz & $63,76 \mathrm{bA}( \pm 16)$ & $49,45 \mathrm{aB}( \pm 22)$ & $115,90 \mathrm{aA}( \pm 26)$ & $91,85 \mathrm{aB}( \pm 17)$ & $299,44 \mathrm{bA}( \pm 31)$ & $282,26 \mathrm{bA}( \pm 39)$ \\
\hline Sem adubo & 70,84 zY (土 19) & $43,72 \mathrm{zZ}( \pm 12)$ & 103,64 yY $( \pm 16)$ & $27,49 \mathrm{zZ}( \pm 5)$ & 424,89 yY $( \pm 63)$ & 312,88 yZ $( \pm 40)$ \\
\hline Com adubo & 86,03 yY $( \pm 17)$ & $59,27 \mathrm{yZ}( \pm 16)$ & 111,16 yY $( \pm 18)$ & 56,98 yZ $( \pm 9)$ & $356,93 \mathrm{zY}( \pm 35)$ & 328,56 yZ $( \pm 45)$ \\
\hline
\end{tabular}

Tabela 4. Volume de óleo extraído por unidade de massa fresca, por tricomas e por unidade de área de folhas jovens e adultas de Mentha x piperita L. var. piperita, cultivadas sob diferentes condições de luz e nutrição do substrato.

Table 4. Volume of extracted oil per fresh mass unit, per trichomes and per leaf area unit of Mentha x piperita L. var. piperita cultivated under different conditions of light and nutrition of the substratum.

\begin{tabular}{|c|c|c|c|c|}
\hline Tratamentos & $\begin{array}{l}\mathrm{mL} 100 \mathrm{~g}^{-1} \\
\text { Folha jovem }\end{array}$ & $\begin{array}{l}\mathrm{mL} 100 \mathrm{~g}^{-1} \\
\text { Folha adulta }\end{array}$ & $\begin{array}{c}\mathrm{mL} 1000 \text { tricomas }^{-1} \\
\text { Folha adulta }\end{array}$ & $\begin{array}{l}\mathrm{mL} \mathrm{cm}^{-2} \\
\text { Folha adulta }\end{array}$ \\
\hline $100 \%$ de luz & 1,64 a A $( \pm 0,20)$ & 1,12 a A $( \pm 0,7)$ & 0,99 a $( \pm 0,09)$ & $0,66 \mathrm{a}( \pm 0,07)$ \\
\hline $70 \%$ de luz & 0,96 a $\mathrm{A}( \pm 0,14)$ & 1,42 a A $( \pm 0,8)$ & $0,99 \mathrm{a}( \pm 0,06)$ & $0,66 \mathrm{a}( \pm 0,10)$ \\
\hline $50 \%$ de luz & 0,90 a $\mathrm{A}( \pm 0,12)$ & 0,96 a $\mathrm{A}( \pm 0,6)$ & $0,74 \mathrm{a}( \pm 0,20)$ & 0,41 a $( \pm 0,10)$ \\
\hline Sem adubo & 1,07 yY $( \pm 0,5$ & 0,49 yY $( \pm 0,06)$ & 0,39 y $( \pm 0,41)$ & $0,244 \mathrm{y}( \pm 0,03)$ \\
\hline Com adubo & 1,64 yY $( \pm 0,2)$ & 1,12 yY $( \pm 0,09)$ & $0,99 \mathrm{y}( \pm 0,09)$ & $0,656 y( \pm 0,07)$ \\
\hline
\end{tabular}

Letras minúsculas (a-b) comparam valores nas colunas pela ANOVA seguida pelo teste de Tukey para os tratamentos de luz e letras (y-z) para os tratamentos com substratos diferentes. Letras maiúsculas (A-B) comparam órgãos jovens e maduros (linhas) para os tratamentos de luz e letras maiúsculas (Y-Z) para os tratamentos de substratos diferentes pelo teste $t$ (Student) where $P<0,05$. ( \pm ) desvio padrão da média / Small letters (a-b) compare values in the columns by ANOVA followed for the test of Tukey, $P<0.05$, for the light treatments and (y-z) for the substrata treatments by $\mathrm{t}$ test, $P<0.05$. Capital letters (A-B) compare young and adult organs (lines) for the light treatments and (Y-Z) for the substrata treatments by $t$ test $, P<0,05$. ( \pm ) Standart deviation.

A quantidade de óleo essencial por unidade de massa fresca também não foi influenciada pela intensidade de luz ou adubação do substrato, confirmando os resultados encontrados em Menthax piperita por Ghosh \& Chatierjee (1976) e Valmorbida (2006) em M. spicata L. por Ghosh \& Chatierjee 1976) e em Mentha x villosa Huds. por Ramos et al. (2005). Embora, luz e nutrição não tenham afetado o rendimento do óleo essencial por unidade de massa, estes fatores podem afetar o rendimento do óleo por planta, causando aumento na área foliar total e biomassa. Em M. piperita o aumento de área foliar e o aumento de biomassa foram fortemente correlacionados $(r=0,96)$, indicando que o aumento em biomassa se deu principalmente pelo aumento em biomassa de folhas. Burbott \& Loomis (1967), Mattos \& Innecco (2002) e Ramos et al. (2005) também encontraram aumento no rendimento de óleos essenciais por planta devido ao aumento em biomassa desta. 
O tamanho das manchas correspondentes ao mentol nas placas cromatográficas (dados não mostrados) foi maior que a de todos os outros componentes dos óleos extraídos das folhas. Isto sugere que o mentol é o componente majoritário dos óleos extraídos de folhas, o que concorda com os dados de Turner et al. (2000) para esta espécie. Aárea das manchas correspondentes ao mentol são maiores nas folhas adultas que nas jovens de plantas adubadas cultivadas a pleno sol (tabela 5). Este dado sugere que adubação associada à alta intensidade de luz pode aumentar a produção de mentol em folhas adultas.

Tabela 5 - Área de manchas correspondentes ao mentol em cromatografia de camada delgada de amostras de óleos essenciais de folhas jovens e adultas de Mentha x piperita $\mathrm{L}$. var. piperita. Plantas cultivadas a $100 \%$ e $50 \%$ de luz com solo adubado e a $100 \%$ de luz com solo não adubado.

Table 5 - Area of stains corresponding to menthol in thin layer chromatograms of essential oils samples from young and adult leaves of Mentha x piperita L. var. piperita. Plants cultivated on $100 \%$ and $50 \%$ light intensity in fertilized substratum and under $100 \%$ light intensity without substratum fertilization.

\begin{tabular}{ccc}
\hline \multirow{2}{*}{ Tratamento } & Folha jovem & Folha adulta \\
\cline { 2 - 3 } & \multicolumn{2}{c}{$\mathrm{cm}^{2}$} \\
\hline $100 \%$ de luz & $2,7 \mathrm{~b}( \pm 0,3)$ & $3,7 \mathrm{a}( \pm 0,2)$ \\
$50 \%$ de luz & $1,7 \mathrm{c}( \pm 0,2)$ & $1,8 \mathrm{c}( \pm 0,1)$ \\
Sem adubo & $2,5 \mathrm{~b}( \pm 0,3)$ & $1,8 \mathrm{c}( \pm 0,2)$ \\
\hline
\end{tabular}

Letras minúsculas comparam valores entre linhas e letras maiúsculas comparam valores entre colunas pela ANOVA multifatorial seguida pelo teste de Tukey, onde $P<0,05$. $( \pm$ ) desvio padrão da média / Small letters compare values in the lines and capital letters in columns by ANOVA followed for the test of Tukey, $P<0.05 .( \pm)$ Standart deviation.

Os resultados aqui encontrados para Menta x piperita indicam a influência da associação entre fertilização do solo e alta intensidade luminosa no aumento da produção de mentol, na diversidade dos componentes do óleo essencial, bem como no aumento da biomassa e área foliar, que indiretamente levam à maior produção de óleos essenciais por planta.

Agradecimentos - Ao PROF/Capes pelo apoio financeiro.

\section{Referências bibliográficas}

BURBOTT, A.J. \& LOOMIS, W.D. 1967. Effects of light and temperature on the monoterpenes of peppermint. Plant Physiology 42:20-28.
CARDOSO, M.G., SHAN, A.Y.K.V., PINTO, J.E.B.P., FILHO, N.D. \& BERTOLUCCI, S.K.V. 2001. Metabólitos secundários vegetais: visão geral química e medicinal. Universidade Federal de Lavras, Lavras.

CASTRO, E.M., PINTO, J.E.B.P., SOARES, A.M., MELO, H.C., BERTOLUCCI, S.K.V., VIEIRA, C.V. \& LIMA JÚNIOR, E.C.L. 2007. Adaptações anatômicas de folhas de Mikania glomerata Sprengel (Asteraceae), em três regiões distintas da planta, em diferentes níveis de sombreamento. Revista Brasileira de Plantas Medicinais 9:8-16.

CASTRO, E.M., PINTO, J.E.B.P., MELO, H.C., SOARES, A.M., ALVARENGA, A.A. \& LIMA JÚNIOR, E.C. 2005. Aspectos anatômicos e fisiológicos de plantas de guaco submetidas a diferentes fotoperíodos. Horticultura Brasileira 23:846-850.

CHAZDON, R.L. \& KAUFMANN, S. 1993. Plasticity of leaf anatomy of two rain forest shrubs in relation to photosynthetic light acclimation. Functional Ecology 7:385-394.

DAVID, E.F.S., BOARO, C.S.F. \& MARQUES, M.O.M. 2006. Rendimento e composição do óleo essencial de Mentha piperita L., cultivada em solução nutritiva com diferentes níveis de fósforo. Revista Brasileira de Plantas Medicinais 8:183-188.

DURIYAPRAPAN, S., BRITTEN, E.J. \& BASFORD, K.E. 1986. The effect of temperature on growth, oil yield and oil quality of japanese mint. Annals of Botany 58: 729-736.

FARMACOPÉIA BRASILEIRA. 2000. 4a ed., parte I, Ateneu, São Paulo, p.2-7.

GHOSH, M.L. \& CHATIERJEE, K. 1976. Effect of N:P: $\mathrm{K}$ fertilizers of growth, development \& essential oil content of Mentha spp. Indian Journal of Experimental Biology 14:366-367.

HUNT, R. 1982. Plant growth curves. The functional approach to plant growth analysis. Edward Arnold, London.

JURIK, T.W., CHABOT, J.F. \& CHABOT, B.F. 1982. Effects of light and nutrients on leaf size, $\mathrm{CO}_{2}$ exchange, and anatomy in wild strawberry (Fragaria virginiana). Plant Physiology 70:1044-1048.

KNETCHT, G.N. \& O'LEARY, J.W. 1972. The effect of light intensity on stomatal density of Phaseolus vulgaris leaves. Botanical Gazette 133:132-134.

LARCHER, W. 2000. Ecofisiologia vegetal. RiMa Artes e Textos, São Carlos.

LIMA, H.R.P., KAPLAN, M.A.C. \& CRUZ, A.V.M. 2003. Influência dos fatores abióticos na produção e variabilidade de terpenóides em plantas. Floresta e Ambiente 10:71-77.

MAFFEI, M., CHIALVA, F. \& SACCO, T. 1989. Glandular trichomes and essential oils in developing peppermint leaves: Variation of peltate trichomes number and terpene distribution within leaves. New Phytologist 111:707-716. 
MATTOS, S.H. \& INNECCO, R. 2002. Idade ideal de corte da Mentha arvensis L. como produtora de óleo essencial e mentol para o Estado do Ceará, Brasil. Revista Brasileira de Plantas Medicidnais 5:15-18.

MEDRI, M.E. \& LLEDRAS, E. 1980. Aspectos da anatomia ecológica de folhas de Hevea brasiliensis Muel. Arg. Acta Amazônica 10:463-493.

MELLO, H.C., CASTRO, E.M., SOARES, A.M., MELO, L.C. \& ALVES, J.D. 2007. Alterações anatômicas e fisiológicas em Setaria anceps Stapf ex Massey e Paspalum paniculatum L. sob condições de déficit hídrico. Hoehnea 34:145-143.

NAVES, V.L,ALVARENGA, A.A. \& OLIVEIRA, L.E.M. 1994. Comportamento estomático de mudas de três espécies florestais submetidas a diferentes níveis de radiação fotossinteticamente ativa. Ciência e Prática 18:408-414.

POORTER, L. 1999. Growth responses of 15 rain-forest tree species to a light gradient: the relative importance of morphological and physiological traits. Ecological Society 13:396-410.

PRASZNA,L.\&BERNÁTH, J. 1993. Correlations between the limited level of nutrition and the essential oil production of peppermint. Acta Horticulturae 344:278-289.

RAMOS, S.J., FERNANDES, L.A., MARQUES, C.C.L., SILVA, D.D., PALMEIRA, C.M. \& MARTINS, E.R. 2005. Produção de matéria seca e óleo essencial de menta sob diferentes doses de fósforo. Revista Brasileira de Plantas Medicinais 8:9-12.

RODRIGUES, C.R., FAQUIN, V., TREVISAN, D., PINTO, J.E.B.P., BERTOLUCCI, S.K.V. \& RODRIGUES, T.M. 2004. Nutrição mineral, crescimento e teor de óleo essencial da menta (Mentha x piperita L.) em solução nutritiva sob diferentes concentrações de fósforo e época de coleta. Horticultura Brasileira 22:573-578.

ROHLOFF, J., DRAGLAND, S., MORDAL, R. \& IVERSEN, T.H. 2005. Effect of harvest time and drying method on biomass production, essential oil yield, and quality of peppermint (Mentha x pipeita L.). Journal of Agricultural and Food Chemistry 53:4143-4148.
SATO, H., YAMADA, K., MIJ, M., HOSOMI, K., OKUYAMA. S., USAWA, M., ISHIKAMA, H. \& ITO, Y. 1996. Production of an interspecific somatic hybrid between peppermint and gingermint. Plant Science 115:101-107.

SILVA, E.A.M. \& ANDERSON, C.E. 1985. Influência da luz no desenvolvimento foliar do feijoeiro (Phaseolus vulgaris L.). Revista Ceres 32:1-11.

SIMÕES, C.M.O. \& SPITZER, V. 2004. Óleos voláteis. In Farmacognosia - da planta ao medicamento (C.M.O. Simões et al., org.). 5a ed. Ed. Universidade/ UFRGS/Ed. da UFSC, Porto Alegre/ Florianópolis, p.467-495.

SOKAL, R.R. \& ROHLF, F.J. 1969. Biometry. Freeman and Company, San Francisco.

STAHL, E. \& SCHILD, W. 1981. Pharmazeutische Biologie Drogenanalyse II: Inhaltsstoffe und Isolierungen. Gustav Fischer Verlag-Stuttgard, New York.

TAVISH, H.M. \& HARRIS, D. 2002. An economic study of essential oil production in the UK: a case study comparing non-UK lavander/lavandin production and peppermint/spearmint production with UK production techniques and costs. For the Government Industry, Forum for Non-Food Crops. The Scotch Parlament, Edinburg.

TURNER, G.W., GERSHENZON, J. \& CROTEAU, R.B. 2000. Distribution of peltate glandular trichomes on developing leaves of peppermint. Plant Physiology 124:655-663.

VALMORBIDA, J., BOARO, C.F.S., MARQUES, M.O.M. \& FERRI, A.F. 2006. Rendimento e composição química de óleos essenciais de Mentha piperita L. cultivada em solução nutritiva com diferentes concentrações de potássio. Revista Brasileira de Plantas Medicinais 8: 56-61.

WILKINSON, H. 1988. The plant surface (mainly leaf). In Anatomy of the dicotyledons (C.R. Metcalfe \& L. Chall, ed.). $2^{\mathrm{a}}$ ed. Oxford University Press, Oxford, p.97-165. 
